\title{
The Spreading Worldwide Obesity Epidemic
}

\author{
Avrahom Aharon Gurwitz
}

Published online: 18 June 2014

(C) Springer Science+Business Media New York 2014

Keywords Childhood and adolescent obesity - Increasing obesity rates in children in Southeastern Europe .

Urban-rural childhood disparities in obesity rates

The article by Genc Burazeri et al. that appears in this issue of the Journal titled Prevalence and demographic correlates of overweight and obesity among children in a transitional Southeastern European population is a very well designed epidemiological study with a remarkable response rate (97\%) for the number of participants involved $(\mathrm{n}=$ $5,810)$ ! The study is well written and makes some very important observations. Of interest is the fact that the obesity rate was lower for girls. This is a bit unusual, but this perhaps could be explained by the younger age of the children and by the fact that they were likely pre-pubescent. After puberty, because of the growth spurt in boys, obesity and overweight fall in boys and increase in girls due to the increased hormonal effects of estrogen. The higher obesity rates in rural versus urban areas is not that surprising. Urban areas have more resources, such as public transportation, but also have limited green spaces such as parks, which limit access for physical activities. In urban areas children are more likely to be eating processed foods from commercial supermarkets rather than whole foods from the field. On the other hand, children in rural areas are more likely to engage in physical activity for daily chores and have much more open spaces to run and play in. Families are also more likely to eat local healthy produce.

Sadly, however, the trend in Albania is the same seen in resource rich countries. As more cheaply processed foods become available in resource poor countries there is an increase of weight gain among the population. This is especially important in children as recent studies have shown that children who are overweight are much more likely to remain overweight their entire lives than people who become overweight later in life. This leads to an increase in diabetes, heart disease, metabolic syndrome in the younger population. Health costs increase dramatically and this puts a strain on the entire healthcare system. In resource poor countries this is especially important as the already fragile healthcare system may collapse with the marked strain obesity puts on it. This important study highlights this crucial point.

A. A. Gurwitz $(\square)$

Holy Redeemer Hospital, Philadelphia, PA, USA

e-mail: Avifs8@gmail.com 